\title{
latrogenic nerve injuries following surgery for paediatric distal humeral epiphyseal fractures - a report of 2 cases
}

\author{
Andrzej Żyluk ${ }^{A 凶}$, Piotr PuchalskiB ${ }^{B}$ Zbigniew Szlosser ${ }^{\mathrm{C}}$ \\ Pomeranian Medical University in Szczecin, Department of General and Hand Surgery, Unii Lubelskiej 1, 71-252 Szczecin, Poland \\ A ORCID: 0000-0002-8299-4525; $\quad{ }^{\text {B }}$ ORCID: 0000-0002-0865-1647; $\quad$ C ORCID: 0000-0002-7334-7932 \\ $\bowtie$ azyluk@hotmail.com
}

\begin{abstract}
Two cases of paediatric radial and ulnar nerve injuries occurring at operative treatment of distal humeral epiphyseal fractures. In one case, the nerve laceration was associated with a defect, requiring reconstruction by nerve grafting. In a 2 nd case, the nerve was compressed and partially damaged by implanting, followed by entrapment in the scar; release of the nerve from
\end{abstract}

the scar resulted in recovery of its function. The importance of being familiar with the nerves' course at the distal end of the humerus, as well as proper and prompt reaction to symptoms of nerve dysfunction occurring immediately after the operation was emphasised.

Keywords: paediatric distal humeral epiphyseal fractures; iatrogenic nerve injury; nerve ultrasound.

\section{INTRODUCTION}

Paediatric distal humeral epiphyseal fractures are relatively common. They occur usually as a consequence of a fall on the elbow or direct blow. These injuries frequently occur in association with intra-articular entrapment of the fracture fragment, elbow dislocation, ulnar or radial nerve damage, and other upper limb fractures. Medial epicondyle fractures often occur as a result of an avulsion force and less frequently due to direct trauma. Non- or minimally displaced condyle fractures can be managed conservatively, but fractures involving epicondyles usually require fixation, due to their unstable avulsion nature (medial epicondyle is an attachment site for flexor digitorum muscles and lateral epicondyle for extensor muscles). We present 2 cases of iatrogenic injuries of radial and ulnar nerves in paediatric patients who were operated on due to fractures involving distal humeral epiphysis and medial epicondyle.

\section{CASE REPORT 1}

A 6-year old boy presented in May 2015 to the department of paediatric orthopaedic surgery with a displaced fracture of the distal humerus, as a consequence of a fall on the elbow (Fig. 1). He was immediately operated on under general anaesthesia. The fracture was reduced and bone fragments were fixed percutaneously with K-wires (Fig. 2). Immediately after the operation, features of complete radial nerve palsy were observed: "dropped" hand and inability of active extension in the wrist and fingers, which were not present at admission. Vascular supply to the hand was normal. In spite of obvious symptoms of radial nerve damage, no diagnostic or therapeutic measures were undertaken: the elbow was immobilized in a plaster slab and the patient was sent home. Three weeks after the operation, the immobilization was removed, K-wires retrieved and the patient was given rehabilitation. As the features of nerve palsy continued, USG and electromyography (EMG) were performed showing discontinuity and severe conduction disturbances in the radial nerve at the elbow, both suggesting serious nerve laceration. In spite of these findings, no intervention was undertaken by the treating team. The child's parents themselves sought medical advice from the authors' institution in November 2015, 6 months following the initial injury. After examination of the patient and the results of USG and electrophysiological studies, the decision to operate immediately was made. Surgery was performed under general anaesthesia and with a tourniquet inflated on the arm. The radial nerve was exposed via lateral incision in the distal arm and cubital fossa. Nerve stumps were found and excised, which resulted in a $4 \mathrm{~cm}$ defect of the nerve. Next, the continuity of the nerve was restored by sural nerve grafting (Fig. 3). The postoperative course was uneventful, but over the next 6 months no symptoms of nerve recovery were observed. In June 2016, 7 months after radial nerve reconstruction, the patient was prepared for tendon transfer to restore wrist and finger extension. Two weeks before the planned date of admission, the parents informed us that they saw a slight extension of the wrist and fingers in the child's hand. The operation was cancelled and over the next 2 months the nerve function recovered almost completely. The patient was seen 15 months after nerve reconstruction showing full wrist and finger extension (Fig. 4). A slight deficit in thumb extension remained, having no effect on normal use of the hand by the child. As clinical examination showed almost complete function of the repaired nerve, no control electrophysiological studies were performed. 


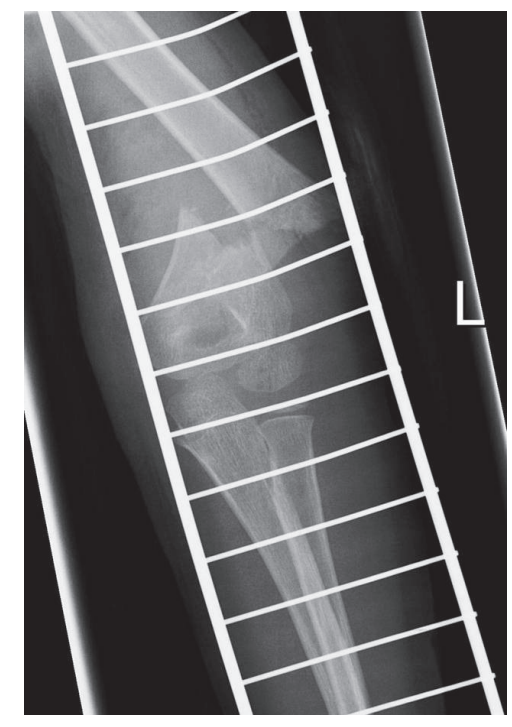

FIGURE 1. Initial X-ray of the fracture of the distal humerus

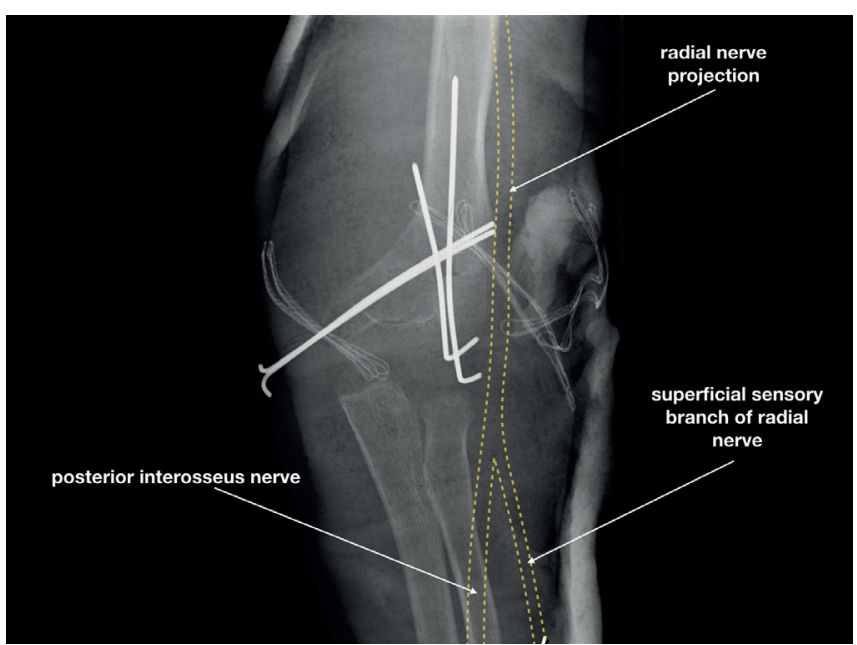

FIGURE 2. Post-operative X-ray showing position of K-wires and course of the radial nerve

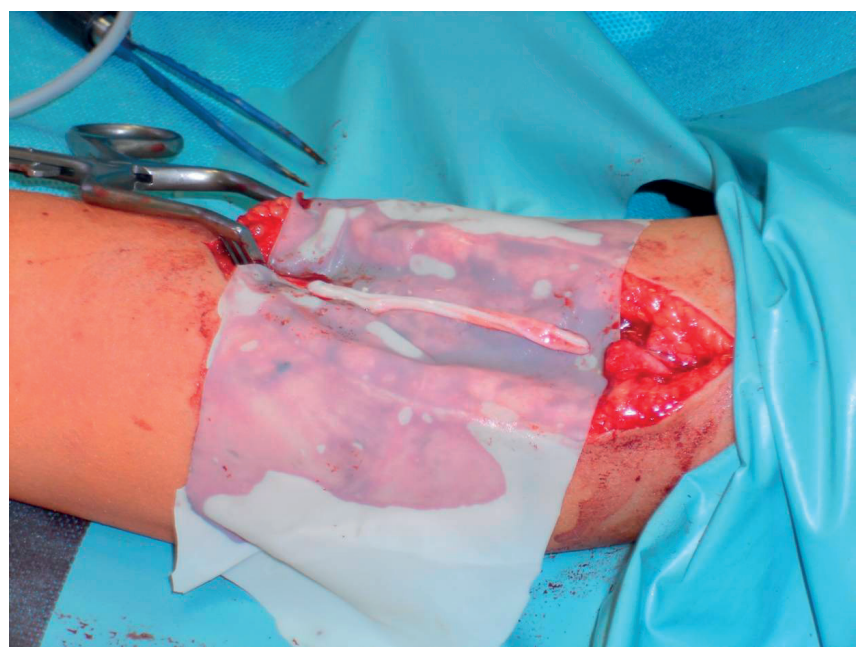

FIGURE 3. Sural nerve grafts ready to be anastomosed with nerve stumps

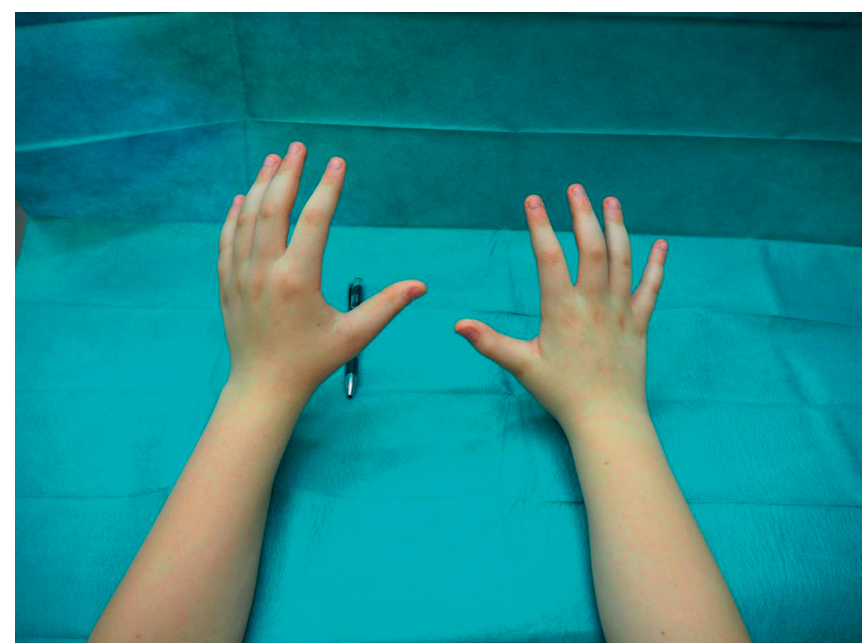

FIGURE 4. Excellent wrist and finger extension 15 months after nerve repair

\section{CASE REPORT 2}

A 7-year old girl sustained a fracture to the medial humeral epicondyle as a consequence of a fall on the elbow (Fig. 5). She was admitted to the department of paediatric orthopaedic surgery and operated on as an emergency. The dislocated medial epicondyle was reduced and fixed percutaneously with $3 \mathrm{~K}$-wires (Fig. 6). In the first postoperative days, features of ulnar nerve dysfunction developed: loss of sensation and incomplete extension in the little and ring fingers (clawing), as well as loss of abduction of the fingers. These symptoms and signs were not present on admission. Unlike the previously reported case, USG was performed promptly, showing swelling and attenuation of the fascicular pattern of the ulnar nerve at the elbow; the nerve's continuity was preserved. Electrophysiological tests showed serious conduction disturbances of the ulnar nerve at the elbow. After removal of the K-wires at 4 weeks, the child was referred to physiotherapy which failed to restore the nerve function over the next 2 months. The child's parents sought medical advice from the authors' institution on their own initiative, 3 months after the fracture and operation. At examination, all features of ulnar nerve palsy were seen and, based on these findings and the results of USG and EMG, we decided to give the patient surgery. The ulnar nerve was exposed via medial incision in the distal arm and elbow. As shown in USG, the continuity of the nerve was preserved, but it was entrapped in scar tissue immediately below the ulnar nerve groove of the humerus, directly at the site of passing of the "ulnar" K-wire (Fig. 6). The nerve was just released from the scar, but not transposed anteriorly. The postoperative course was uneventful and the 1st symptoms of nerve recovery were observed 1 month after the operation: a slight improvement in sensation and withdrawal of clawing in the little and ring fingers. At 4 months follow-up, the patient presented normal function of the ulnar nerve and, therefore, no control electrophysiological examination was performed. 


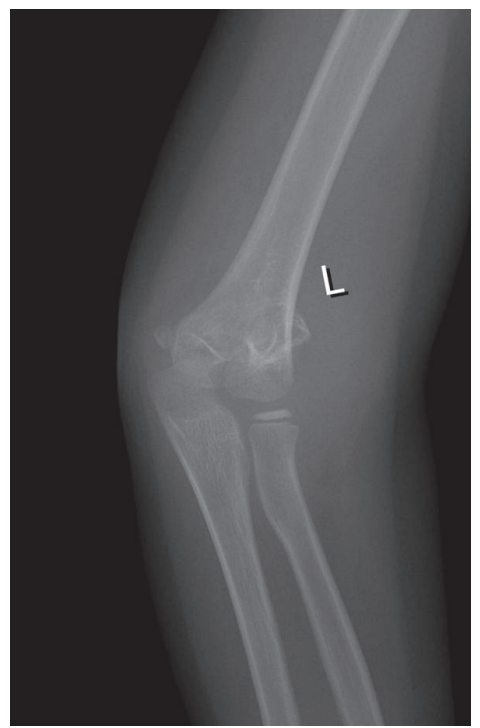

FIGURE 5. Initial X-ray of the fracture of the medial epicondyle

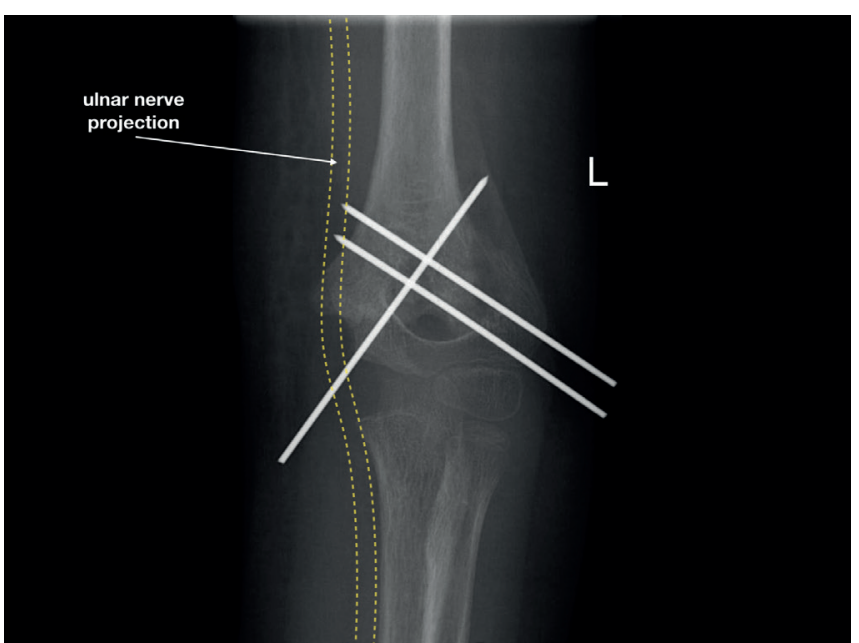

FIGURE 6. Post-operative X-ray showing position of K-wires and course of the ulnar nerve

\section{DISCUSSION}

Nerve injuries concomitant with paediatric distal humeral epiphyseal fractures are not frequent. We found only single reports in literature. Non- or malunion resulting in functional dysfunction, restriction of range of motion and valgus attitude of the elbow are more frequently described $[1,2,3]$. Both of the nerve injuries reported in this study were iatrogenic and occurred at operative treatment of distal humerus fractures. The cause of excessive radial nerve laceration in the 1st case is difficult to explain. Dislocation of the fracture, although serious, should (theoretically) not cause this, but, at the very most transient dysfunction of a neuropraxial nature. More likely the laceration occurred during fixation of the fracture with K-wires. One can speculate that the surgeons wounded the nerve when repeating introduction of the wires several times to obtain the perfect position. In the 2nd case, the K-wire was placed immediately by the ulnar nerve, causing its partial injury followed by entrapment in the scar. Release of the nerve from the scar resulted in full functional recovery.

The surgeons' activity in the 1st case could be called malpractice, as it was not consistent with current medical knowledge and not sufficiently careful (accurate). Ignoring sonographic, electrophysiological and clinical evidence of serious radial nerve damage and decision on conservative treatment was a mistake. In this situation, the only rational solution was to operate on the patient as soon as possible. In such a large nerve defect, its reconstruction by sural nerve grafting was an optimal - and eventually successful - procedure. Outcomes of nerve repair at the arm level, particularly delayed and requiring grafting are not fully predictable. Although the prognosis is better in children, an almost full recovery of nerve function in such a big defect can be considered a success.

We found several reports in literature presenting ulnar nerve injuries associated with elbow dislocation and fracture of the medial epicondyle in 10-12-year-old boys [1, 2, 3, $4,5,6,7,8,9]$. In most of these cases, epicondylar fracture was initially not diagnosed, resulting in its displacement and compression of the ulnar nerve. All these patients were operated on 6-8 months after the initial fractures by resection of non-united epicondyle and ulnar nerve release ( 2 cases) or by re-fixation of the epicondyle followed by anterior transposition of the nerve ( 1 case). The results were satisfactory in all cases $[1,2,3]$. Babal et al. in their meta-analysis reported that the most common nerve involved in iatrogenic neuropraxia after fixation of supracondylar humeral fractures was the ulnar (3.2\%) followed by the median (1.8\%) and radial (1.6\%) nerves. These authors grouped the iatrogenic neuropraxias by pin configuration and found a rate of $3-4 \%$ in patients who had lateral-only pinning and $4.1 \%$ in those who had treated with medial pinning [4]. In 3 other articles, the authors suggest that nerve injuries associated with supracondylar fractures in most cases recover spontaneously and well, and only some, with complete lesions in EMG or USG and those in whom the anticipated clinical recovery does not occur require operative exploration. The average rate of iatrogenic nerve injuries associated with closed fixation of the fractures is estimated at $3 \%$. A trend toward the more severely displaced fractures having a higher incidence of postoperative neuropraxia was observed $[5,6,7]$.

In one Polish study, nerve damage was found in 22 of 220 paediatric patients with displaced supracondylar fractures, most commonly the median (15/22), followed by the ulnar $(6 / 22)$ and radial $(1 / 22)$ nerves. In 20 patients (91\%) symptoms of nerve damage resolved spontaneously and in 2 cases ( $9 \%$ ), an operation was necessary consisting of release of entrapped nerves form the scar. This study, however, does not provide information on how many fractures were treated operatively and, therefore, how many nerve injuries were true iatrogenic [8]. 
These injuries are seen in adults as a consequence of open fixation of displaced fractures of distal humerus. Classen et al. reported traumatic radial nerve palsy that had occurred in 66 of 325 diaphyseal humeral fractures $(20 \%)$ and iatrogenic laceration or dysfunction of the radial nerve occurring in 18 of 259 patients (7\%) [9]. The risk of nerve damage was found the highest at lateral approach to the distal humerus and was usually caused by traction, but sometimes due to pressure from a retractor, the exposure, or damage from a drill or implant. All iatrogenic radial nerve palsies were complete motor palsies. In 5 of these 18 cases nerve laceration required repair with sural nerve grafting with a good final outcome [9].

Both reported cases are interesting due to their rarity. Both were caused by negligence and inaccuracy during closed fixation of the fractures around the distal part of the humerus, the area where the radial and ulnar nerves pass close to the bone. It seems that surgeons should be aware of the anatomical location of the nerves and try to avoid them when introducing K-wires. The 2nd message drawn from these reports concerns inadequate reaction to obvious features of nerve damage. This is particularly evident in the 1st case. Ultrasonography examination is sensitive enough to show nerve status (compression, oedema or discontinuity) in the case of post-traumatic or post-operative dysfunction. Diagnosis of nerve laceration is an absolute indication for surgical exploration and repair, and any delay in making this decision must be considered malpractice.

\section{REFERENCES}

1. El-Sobky TA, Haleem JF, Sakr HM, Aly AS. A neglected markedly displaced medial epicondyle fracture with simultaneous ulnar nerve palsy in an adolescent. Clin Orthop Surg 2017;9(4):542-6.

2. Lima S, Correia JF, Ribeiro RP, Martins RM, Alegrete N, Coutinho J, et al. A rare case of elbow dislocation associated with unrecognized fracture of medial epicondyle and delayed ulnar neuropathy in pediatric age. J Shoulder Elbow Surg 2013;22(3):e9-11.

3. Haflah NH, Ibrahim S, Sapuan J, Abdullah S. An elbow dislocation in a child with missed medial epicondyle fracture and late ulnar nerve palsy. J Pediatr Orthop 2010;19(5):459-61.

4. Babal JC, Mehlman CT, Klein G. Nerve injuries associated with pediatric supracondylar humeral fractures: a meta-analysis. J Pediatr Orthop 2010;30(3):253-63.

5. Joiner ER, Skaggs DL, Arkader A, Andras LM, Lightdale-Miric NR, Pace JL, et al. Iatrogenic nerve injuries in the treatment of supracondylar humeral fractures: are we really just missing nerve injuries on preoperative examination? J Pediatr Orthop 2014;34(4):388-92.

6. Guner S, Guven N, Karadas S, Ceylan MF, Turktas U, Gokalp NA, et al. Iatrogenic or fracture-related nerve injuries in supracondylar humerus fracture: is treatment necessary for nerve injury? Eur Rev Med Pharmacol Sci 2013;17(6):815-9.

7. Ramachandran M, Birch R, Eastwood DM. Clinical outcome of nerve injuries associated with supracondylar fractures of the humerus in children: the experience of a specialist referral centre. J Bone Joint Surg Br 2006;88(1):90-4

8. Tomaszewski R, Wozowicz A, Wysocka-Wojakiewicz P. Analysis of early neurovascular complications of pediatric supracondylar humeral fractures: a long term observation. Biomed Res Int 2017;2017:2803790. doi: 10.1155/2017/2803790.

9. Claessen FM, Peters RM, Verbeek DO, Helfet DL, Ring D. Factors associated with radial nerve palsy after operative treatment of diaphyseal humeral shaft fractures. J Shoulder Elbow Surg 2015;24(11):e307-11. 